\title{
Can an Enhanced Recovery After Surgery (ERAS) programme improve colorectal cancer outcomes in South Africa?
}

\author{
R L Oodit ${ }^{1}$, O Ljungqvist ${ }^{2}$, J Moodley ${ }^{3}$ \\ ${ }^{1}$ Department of Surgery, Faculty of Health Sciences University of Cape Town and Groote Schuur Hospital Observatory 7925, \\ South Africa. Matley and Partners, Constantiaberg Hospital, Cape Town \\ ${ }^{2}$ Professor of Surgery, Department of Surgery, Faculty of Medicine and Health, Örebro University, Örebro, Sweden, Örebro \\ ${ }^{3}$ Cancer Research Initiative, Faculty of Health Sciences, University of Cape Town, Observatory 7925, South Africa. Women's \\ Health Research Unit, School of Public Health \& Family Medicine, Faculty of Health Sciences, University of Cape Town
}

Corresponding author: Ravi Oodit (ravio@surgcare.co.za)

\section{Introduction}

Colorectal cancer (CRC) is the third most common cancer worldwide and the fourth most common cause of cancer related deaths. ${ }^{1}$ It is estimated that $\mathrm{CRC}$ is amongst the top five malignancies in South Africa (SA) with an age standardised incidence rate of 10.2 and 6.1 per 100000 for males and females respectively. ${ }^{2}$ The incidence is projected to increase in South Africa as a result of ageing, a growing population and an increase in prevalence of risk factors. ${ }^{1}$

Surgery is central to the treatment of patients with CRC. Treatment includes resection for early-stage CRC and a combination of resection and adjuvant chemotherapy for latestage CRC. The management of metastatic disease includes resection of liver and or lung metastases. Colonic stenting is used as an adjunct to surgery and has a role in palliative care.

Traditionally colorectal surgery is associated with a complication rate of $20 \%$ to $40 \%$ and a hospital stay of 7 to 10 days. ${ }^{3,4,5}$ Complication rates and length of stay vary significantly between and within countries. Short- and long-term mortality is significantly higher among patients who develop postoperative complications. ${ }^{6}$ Conventional perioperative care after colorectal surgery in SA, as in many parts of the world, is often not evidence-based and frequently lacks a patient-centred, integrated team approach.

The Enhanced Recovery After Surgery (ERAS) care pathway is patient centred, multi-disciplinary team approach to perioperative care. In countries where ERAS has been implemented for patients undergoing elective colorectal surgery postoperative complications and hospital stay has been reduced by $40-50 \% .^{3,4}$ and costs by $10-20 \% .^{7,8}$ Further a decrease in nursing work load has also been achieved. ${ }^{9} \mathrm{~A}$ recent single centre retrospective study from Sweden reported that the risk of 5-year cancer-specific mortality rate was decreased by $42 \%$ when compliance with ERAS guidelines was above $70 \% .^{10}$ This is the only study reporting on compliance and cancer mortality rate. Randomised controlled trials are required to confirm these findings.

\section{The ERAS Care System}

Professors Olle Ljundgvists (Orebro University, Sweden) and Ken Fearon (Edinburg University, Scotland) initiated the ERAS Study Group in 2001, which later evolved into the ERAS Society. ${ }^{11}$ The Society established the ERAS Care System with 3 main components: ${ }^{12}$ (a) Evidence based management guidelines; (b) an implementation program and (c) a monitoring and evaluation system. These are described briefly below:

(a). The ERAS management guidelines address pre-, intra- and post-operative practice. ${ }^{12}$ and are outline in Table 1. The guidelines focus on reducing the peri-operative pathophysiological catabolic stress response and immune suppression. ${ }^{13}$

A meta-analysis of single and multi-centre studies have shown that as compliance to the ERAS guidelines improve, complications are reduced. ${ }^{14,15,16}$ Centre's with a $90 \%$ compliance rate had complication rates of less than $20 \%$. Those with a compliance rate of $50 \%$ or less had a complication rate of $50 \%$.

The management guidelines (Table1) are based on best available evidence and use the Grading of Recommendations, Assessment, Development and Evaluation (GRADE) system. ${ }^{17}$ The GRADE system is used by many international organizations e.g. Cochrane, the National Institute for Health and Clinical Excellence (NICE), UpToDate ${ }^{\circledR}$ and the World Health Organization (WHO), to make recommendations 
Table 1: Key elements of the ERAS management guidelines

\begin{tabular}{|c|c|c|}
\hline Preoperative & Intraoperative & Postoperative \\
\hline Pre admission counselling & Selective,mid-thoracic epidural & Selective, mid-thoracic epidural \\
\hline Carbohydrate preload & Short acting anaesthetic drugs & Early enteral feeding \\
\hline Antibiotic prophylaxis & Maintain normothermia & Early mobilization \\
\hline Thrombo-prophylaxis & Nausea \& Vomiting prophylaxis & Prevent nausea and vomiting \\
\hline Selective use of bowel preparation & Avoid long acting opioids & Avoid opioid analgesia \\
\hline No prolonged fasting & Avoid fluid overload & Avoid fluid overload \\
\hline \multirow[t]{3}{*}{ No premedication } & Avoid drains & Early removal of catheter \\
\hline & No nasogastric tube & No nasogastric tube \\
\hline & & $\begin{array}{l}\text { Reduce ileus: chewing gum } \\
\text { Magnesium }\end{array}$ \\
\hline
\end{tabular}

Table 2: GRADE of evidence and recommendation for the ERAS guidelines

\begin{tabular}{|c|c|c|}
\hline & Evidence & Recommendation \\
\hline Pre admission Counselling & Low & Strong \\
\hline Carbohydrate preload & Moderate & Strong \\
\hline Antibiotic prophylaxis & High & Strong \\
\hline Thrombo-prophylaxis & High & Strong \\
\hline Selective use of bowel preparation & High & Strong \\
\hline No prolonged fasting & Moderate & Strong \\
\hline No premedication & High & Strong \\
\hline Selective mid-thoracic epidural & High & Strong \\
\hline Maintain normothermia & High & Strong \\
\hline Nausea and vomiting prophylaxis & Low & Strong \\
\hline Avoid long acting opioid's & High & Strong \\
\hline Avoid fluid overload & High & Strong \\
\hline No nasogastric tube & High & Strong \\
\hline Avoid drains & High & Strong \\
\hline Early enteral feeding & High & Strong \\
\hline Early mobilization & Low & Strong \\
\hline Early removal of urinary catheter & Low & Strong \\
\hline \multicolumn{3}{|l|}{ Stimulation of gut motility } \\
\hline Chewing gum & Moderate & Strong \\
\hline Magnesium & Low & Weak \\
\hline
\end{tabular}

for a variety of conditions. The GRADE system classifies the quality of evidence as high, moderate, low or very low and recommendations are graded weak or strong. Using the GRADE system, 20 key elements of ERAS guidelines have been graded as high (13/20), moderate (3/20) and low (4/20). The majority (19/20) of recommendations have been classified as strong (Table 2) (b). The ERAS Implementation program is focused on building a well-functioning integrated multidisciplinary team that is able to effect the evidence-based guidelines. The program adopts change management principles and includes a series of seminars and action periods. It is run over a period of 10 months. ${ }^{11}$ The team is centred around the patient and their families. Team members includes the surgeon, anaesthetist, 
ERAS nurse coordinator, nursing and theatre teams, physiotherapist, dietician, physician, data-capturer, hospital management and administrators. A key member of the team is the ERAS nurse coordinator who accompanies the patient from the time of diagnosis to their discharge. The nurse coordinator is responsible for the pre-operative counselling, discharge planning, postoperative care and plays a crucial role in teaching and training.

(c). The ERAS monitoring and evaluation system is an integral part of the implementation program as it allows the teams to continuously monitor their compliance to the guidelines, measure their outcomes and effect change ${ }^{8}$. This is based on the Deming Plan-Do-Study-Act (PDSA) cycle. ${ }^{18}$ The system is a web based, real time database that is also designed for research allowing centres to conduct locally and internationally relevant research and to benchmark their outcomes and compliance against other ERAS centres.

\section{Discussion: Implementation of ERAS in South Africa}

The ERAS Care System has been integrated into perioperative care in high income countries: Sweden, Norway, Switzerland, Germany the United Kingdom, Denmark, Spain, New Zealand, Canada and continues to extend its footprint globally. SA, a middle income country, has embarked on implementing the ERAS program. The South African Society of Endoscopic Surgeons (SASES), The Association of Surgeons of South Africa (ASSSA) and the South African Perioperative Research Group (SAPORG) has placed the Implementation of the ERAS Care System a priority in SA. SAPORG has this as one of its top ten perioperative research priorities. ${ }^{19}$ However, implementation of ERAS in SA will require consideration of the very different health care context compared that of high income settings. Specifically, implementation will need to take into account the nutritional status of the population, the high prevalence of HIV, the limited access to health care and the resource-constrained health system.

\section{Nutritional status of the population}

Obesity adds to the complexity of surgery and perioperative care. It is also associated with increased comorbidities, higher complication rates and longer length of stay. Malnourished patients have significantly higher morbidity and mortality, a longer length of stay (LOS) and increased hospital costs ${ }^{20,21,22}$ Improving the patient's nutritional status prior to surgery is associated with improved outcomes.

In SA malnutrition and obesity are significant public health problems. SA has the highest prevalence of obesity in subSaharan Africa. The SANHANES -1 study reports that $65 \%$ of adult females and $30 \%$ of adult males are either overweight or obese. ${ }^{23}$ In SA, $26 \%$ of the population are food insecure and $4-11 \%$ of the adult population are malnourished. ${ }^{23}$ Given the high prevalence of obesity and malnutrition in SA, the benefits of the ERAS program may not be fully realized if patients are not nutritionally optimized preoperatively. This could be difficult to achieve in LMIC.

Routine nutritional assessment and support, a key element of the ERAS program, is not traditional practice in SA.
To address this, dieticians will need to play a larger role in assessing, monitoring and supporting patients. The current shortage of dieticians in the SA health system will need to be addressed. ${ }^{24}$ In addition other health care professionals will need training and education on the importance of preoperative nutritional assessment and optimization. Funding will also be required for appropriate nutritional support.

\section{$H I V$ prevalence in $S A$}

SA has the largest HIV epidemic in the world, with an estimated 7.0 million people living with HIV/AIDS. ${ }^{25}$ There is conflicting and limited evidence of the impact of HIV status on postoperative patient outcomes following elective colorectal surgery in SA. Cacala et al., in a study conducted in Kwa-Zulu Natal, reported no difference in postoperative outcomes in $350 \mathrm{HIV}$ positive patients irrespective of CD4 counts when compared to HIV negative patients. However, the study had few colorectal cancer patients and was under powered to address the association between HIV and postoperative colorectal surgery outcomes. ${ }^{26}$ A second study, the South African Surgical Outcomes Study (SASOS) found that HIV infection had no impact on hospital mortality. However, complication was not measured and the study included a variety of surgical procedures, of which $10.2 \%$ were for colorectal surgery. ${ }^{27}$ In contrast, a recent large population based study from China, reported (as an abstract) significantly higher complication rates and a five-fold risk of 30-day mortality in patients with HIV infection undergoing major surgery compared to those that were HIV negative. ${ }^{28}$ However these findings may not be transferable to SA, as HIV acquisition was mostly due to substance abuse, whereas in SA HIV is mostly sexually transmitted. Further local studies are needed to determine the impact of HIV on postoperative outcomes in SA.

If $\mathrm{HIV}$ is associated with poor postoperative outcomes, the benefits of the ERAS program may not be achieved. The ERAS guidelines will need to be revised to take into account the HIV status of patients. This is likely to include routine HIV testing and adequate perioperative antibiotic prophylaxis.

\section{Access to health care}

Implementation of the ERAS program has resulted in patients being discharged earlier from hospital. One of the major concerns with early discharge is the delayed presentation of complications, especially that of an anastomotic leak. An anastomotic leak occurs in $1-30 \%$ of patients and is associated with significant morbidity, mortality (6 -22\%), repeat surgery, longer length of hospital stay, increased costs and poor long term cancer outcomes. ${ }^{29}$ It is the leading cause of postoperative mortality after colorectal surgery. ${ }^{29}$ Early diagnosis and intervention is the key to better outcomes in this group of patients.

Early discharge of postoperative patients might not be as easily achieved in SA as in high income countries. In SA patients attending public sector health services have limited access to transport and health care facilities. Hence surgeons might be hesitant to discharge patients earlier than traditionally done. In additional patients who develop complications at home are likely to present late. Although access to care is better in the private sector, health care 
professionals in solo practice are not always available after hours. The benefits of early discharge with ERAS will not be realized and patient care could be compromised if discharge occurs without adequate support systems in place. Solutions will need to be identified and implemented prior to commencement of the ERAS care pathway otherwise. These could include: preoperative discharge planning to identify and address any barriers to early discharge; group private practise; a check list for alarm symptoms for patients on discharge; a single on-call telephone number that gives patients and their families immediate access to the managing team; daily calls to the patient following discharge; home visits by community health care workers; and the use of home monitoring and step down facilities.

\section{Economic implications}

Significant resources are required to implement the program. Costs include salaries for the ERAS nurse coordinator, datacapturer and administrator, education, research and training, regular team meetings, nutritional support, computer hardware and software and database management. International data show that ERAS results in a cost saving of $10 \%-20 \%{ }^{8,9}$ A local cost benefit analysis will need to be conducted to guide implementation of ERAS in SA.

\section{Conclusion}

Implementation of the ERAS Care system in SA could provide a platform to improve patient outcomes, improve service efficiency, reduce hospital bed days and improve the use of limited resources. However, implementation needs to be carefully planned to take into consideration the health care system and contextual challenges.

\section{REFERENCES}

1. International Agency for Research on Cancer. Globocan 2012: Estimated cancer incidence, mortality and prevalence worldwide in 2012. 2016. http:// globocan.iarc.fr. (accessed Nov 2016)

2. National Cancer Registry. Cancer in South Africa 2011 Full Report National Cancer Registry. 2016.www.nioh.ac.za. (accessed Nov 2016).

3. Greco M, Capretti G, Beretta L, Gemma M, Pecorelli N, Braga $\mathrm{M}$. Enhanced recovery program in colorectal surgery: a metaanalysis of randomized controlled trials World J Surg. 2014 Jun;38(6):1531-41. 2416-8.

4. Varadhan KK, Neal KR, Dejong CH, Fearon KC, Ljungqvist O, Lobo DN. The enhanced recovery after surgery (ERAS) pathway for patients undergoing major elective open colorectal surgery: a meta-analysis of randomized controlled trials. Clinical nutrition 2010;29(4):434-440.

5. Bokey EL1, Chapuis PH, Fung C, Hughes WJ, Koorey SG, Brewer D,Newland R Postoperative morbidity and mortality following resection of the colon and rectum for cancer. Dis Colon Rectum. 1995 May;38(5):480-6; discussion 486-7.

6. Khuri SF, Henderson WG, DePalma RG, Mosca C, Healey NA, Kumbhani DJ. Determinants of long-term survival after major surgery and the adverse effect of postoperative complications. Ann Surg 2005;242(3):326-343

7. Sammour T, Zargar-Shoshtari K, Bhat A, Kahokehr A, Hill AG. A programme of Enhanced Recovery After Surgery (ERAS) is a cost-effective intervention in elective colonic surgery. The New Zealand Medical Journal 2010;123(1319).

8. Lee L, Mata J, Ghitulescu GA, Boutros M, Charlebois P, Stein $B$, et al. Cost-effectiveness of Enhanced Recovery Versus
Conventional Perioperative Management for Colorectal Surgery. Ann Surg 2015;262(6):1026-1033.

9. Hübner M, Addor V, Slieker J, Griesser A, Lécureux E, Blanc $\mathrm{C}$, et al. The impact of an enhanced recovery pathway on nursing workload: A retrospective cohort study. International Journal of Surgery 2015;24:45-50.

10. Gustafsson UO, Oppelstrup H, Thorell A, Nygren J, Ljungqvist $\mathrm{O}$. Adherence to the eras protocol is associated with 5-year survival after colorectal cancer surgery: a retrospective cohort study. World J Surg 2016;40(7):1741-1747.

11. ERAS Enhanced Recovery After Surgery. 2016. http:// erassociety.org/. (accessed Nov 2016).

12. Nygren J, Thacker J, Carli F, Fearon K, Norderval S, Lobo D, et al. Guidelines for Perioperative Care in Elective Rectal/Pelvic Surgery: Enhanced Recovery After Surgery (ERAS Oे) Society Recommendations. World J Surg 2013;37:285-305.

13. Kehlet H. The surgical stress response: should it be prevented? Can J Surg 1991;34(6):565-567.

14. Gustafsson UO, Hausel J, Thorell A, Ljungqvist O, Soop M, Nygren J. Adherence to the enhanced recovery after surgery protocol and outcomes after colorectal cancer surgery. Archives of Surgery 2011;146(5):571-577.

15. The ERAS Compliance Group The Impact of Enhanced Recovery Protocol Compliance on Elective Colorectal Cancer Resection: Results From an International Registry Annals of Surgery: June 2015 - Volume 261 - Issue 6 - p 1153- 1159-1159

16. Gustafsson UO, Scott MJ, Schwenk W, et al. Guidelines for perioperative care in elective colonic surgery: Enhanced Recovery After Surgery (ERAS) Society recommendations. World J Surg 2013;37(2):259-84.

17. Guyatt GH, Oxman AD, Vist GE, Kunz R, Falck-Ytter Y, Alonso-Coello P et al (2008) GRADE: an emerging consensus on rating quality of evidence and strength of recommendations. BMJ 336(7650):924-926

18. Deming $\mathrm{W}$ editor. The New Economics for Industry, Government, Education. Cambridge: Massachusetts Institute of Technology Center for Advanced Engineering Study; 1993.

19. Biccard BM, Alphonsus CS, Bishop DG, et al. National priorities for perioperative research in South Africa. S Afr Med J 2016;106(5):485-8. doi: 10.7196/SAMJ.2016.v106i5.10269

20. Correia MIT, Waitzberg DL. The impact of malnutrition on morbidity, mortality, length of hospital stay and costs evaluated through a multivariate model analysis. Clinical nutrition 2003;22(3):235-239.

21. Naber TH, Schermer T, de Bree A, Nusteling K, Eggink L, Kruimel JW, et al. Prevalence of malnutrition in nonsurgical hospitalized patients and its association with disease complications. Am J Clin Nutr 1997;66(5):1232-1239.

22. Norman K, Pichard C, Lochs H, Pirlich M. Prognostic impact of disease-related malnutrition. Clinical nutrition 2008;27(1):5-15.

23. Shisana O, Labadarios D, Rehle T, Simbayi L, Zuma K. South African National Health and Nutrition Examination Survey (SANHANES-1). 2013. Cape Town HSRC Press:135-144

24. Steyn N, Mbhenyane Workforce development in South Africa with a focus on public health nutrition Public Health Nutrition: 11(8), 792-800

25. UNAIDS. 2016. www.unaids.org. (accessed Nov 2016).

26. Čačala S, Mafana E, Thomson S, Smith A. Prevalence of HIV status and CD4 counts in a surgical cohort: their relationship to clinical outcome. The Annals of The Royal College of Surgeons of England 2006;88(1):46-51.

27. B M Biccard, T E Madiba, on behalf of the South African Surgical Outcomes Study investigators. The South African Surgical Outcomes Study: A 7-day prospective observational cohort study S Afr Med J 2015;105(6):465-475

28. Chen T, Lin Y, Liao C. Postoperative adverse outcomes after major surgery in HIV-infected patients: a nationwide matched cohort study: 1AP2. European Journal of Anaesthesiology 2014;31:7.

29. Daams F, Luyer M, Lange J Colorectal anastomotic leakage: Aspects of prevention, detection and treatment World J Gastroenterology. 2013 Apr 21; 19(15): 2293 -2297. 